\section{Nurse-led formal health review for immunosuppressed patients with uveitis}

\begin{abstract}
Purpose To describe and analyse a method of detailed formal health review for patients with sight-threatening uveitis using oral immunosuppression.

Methods Prospective clinical study of 37 patients.

Results Treatment side effects were absent or tolerable in 31 patients $(84 \%)$ but 34 significant treatment side effects or other significant clinical findings were newly identified. Ten patients underwent a treatment change including three who transferred to a different immunosuppressive.

Conclusions Formal health review has proved useful in identifying treatment side effects previously not volunteered by patients. A modified form is to be embedded within our clinic management system. We recommend such reviews to enhance drug safety in

patients with sight-threatening chronic uveitis using oral immunosuppressives.

Eye (2009) 23, 1400-1403; doi:10.1038/eye.2008.270;

published online 26 September 2008
\end{abstract}

The Royal Eye Hospital, Manchester, Lancashire, UK

Correspondence: NP Jones, Manchester Royal Eye Hospital,

Oxford Road,

Manchester, Lancashire

$\mathrm{M} 13 \mathrm{9WH}$

UK

Tel: + 0161276 1234;

Fax: +01612726618.

E-mail: nicholas.jones@

cmmc.nhs.uk

Received: 19 September 2007

Accepted in revised form: 29 July 2008

Published online:

26 September 2008

The authors have no financial or other interest in any proprietary product or medication discussed in this article
Keywords: uveitis; immunosuppression; steroid; monitoring; safety

\section{Introduction}

Patients with sight-threatening uveitis may require long-term treatment with combined oral steroid and immunosuppression. The adverse effects of these treatments are well-reported. ${ }^{1,2}$ Stringent safety mechanisms are necessary and this may require detailed management protocols. To enhance adherence to our written management protocols, we trialled a nurse-led formal health review system and report our results and conclusions.
NP Jones and M Pickford

\section{Patients and methods}

The Manchester Uveitis Clinic offers a tertiary referral service for patients with sightthreatening uveitis. Oral immunosuppression and steroid treatment are frequently used and managed entirely within the clinic according to the protocols which include instructions on haematological monitoring, dosage, and management of drug-induced complications. Patients are provided with comprehensive verbal and written advice on drug safety. Those using both oral steroid and immunosuppressives require complex monitoring, which may be difficult to guarantee at every visit within a busy clinic setting.

One of us (NPJ) devised a comprehensive formal health review (FHR) protocol (see Supplementary Figure), which was performed by interview and examination at an arranged appointment by prior agreement with the patient. The interview (taking about $30 \mathrm{~min}$ ) was carried out by MP, a uveitis nurse practitioner, the results being recorded on a standardised form. MP had undergone extensive training in the management of immunosuppression and its complications, both personally by NPJ and by attendance at other nurse-led clinics including renal and rheumatology clinics. The patient was then examined and interviewed by NPJ, and after discussion a medium-term management plan was confirmed.

Data included drug dosages and duration of treatment, and a review of all medications to exclude possible interactions. Drug-specific sections were then completed, including a detailed assessment of possible symptomatic side effects and toxicity. For prednisolone, this included an assessment of changes to skin and habitus, a measurement of total weight gain and change in blood pressure and the identification of steroid-induced diabetes; also, a detailed assessment of bone health was made, including the identification of risk factors for osteoporosis, 
the use of bone-protective medication including calcium and vitamin D supplementation and bisphosphonates, a review of bone densitometry results and decisions on change in management. Potential drug-induced side effects were noted for immunosuppressives including azathioprine, mycophenolate mofetil, and cyclosporin.

A history of infections if any, while on immunosuppression, was taken. Confirmation of previous immunisations to measles and tuberculosis, and of exposure to varicella, was made. A discussion on the low risk of malignancy was undertaken, with an information pamphlet made available to the patient. Regular self-examination was described and encouraged.

Cardiovascular risk was assessed, including the possible drug-induced risks of systemic hypertension, obesity, diabetes and hypercholesterolaemia, and the possible intrinsic risks of some forms of uveitis. The coronary risk was assessed using the British National Formulary cardiovascular risk prediction charts.

Recommendations were made on diet, exercise, alcohol, and smoking. Review by the general practitioner was recommended if necessary.

A full review of ophthalmic status was performed, and the patients's status was summarised by three criteria; first the maintenance of target acuity; second the control of intraocular inflammation; and third the tolerability of side effects. An appropriate medium-term treatment plan was devised and clinic review was arranged.

\section{Results}

A total of 37 patients underwent FHR. The diagnoses are shown in Table 1 and the medications used in Table 2. Twenty-eight patients were using prednisolone, at a mean dose of $8.7 \mathrm{mg} /$ day (range $2-20 \mathrm{mg} /$ day) and for a mean duration of 67 months (range 16 months to 28 years). All patients were using one or more oral immunosuppressives. Azathioprine was most commonly used (18 patients, mean dose $100 \mathrm{mg} /$ day, mean duration

Table 1 The uveitis diagnosis for the reviewed patients

\begin{tabular}{lc}
\hline Diagnosis & Number \\
\hline Behçet's disease & 12 \\
Idiopathic chronic panuveitis & 10 \\
Intermediate uveitis & 4 \\
Sarcoidosis & 2 \\
Scleritis & 2 \\
Birdshot retinochoroidopathy & 1 \\
Geographic choroidopathy & 1 \\
Psoriatic arthropathy & 1 \\
Reiter's disease & 1 \\
Primary idiopathic retinal vasculitis & 1 \\
Sympathetic uveitis & 1 \\
Vogt-Koyanagi-Harada disease & 1 \\
\hline
\end{tabular}

24 months) followed by ciclosporin (16, mean dose $2.9 \mathrm{mg} / \mathrm{kg} /$ day, mean duration 44 months) and mycophenolate $(8$, mean dose $2 \mathrm{~g}$ /day, mean duration 18 months). Six patients were using two immunosuppressives.

There were no previously unrevealed drug interactions. Some treatment side effects were newly reported and notably included dermatological side effects (total 10 patients), including steroid-induced striae, steroid- and ciclosporin-induced hirsutism, steroid-induced acne, and ciclosporin-induced warts. Recent infections (total six patients) included antimicrobial treatment including three dental abscesses, one episode of varicella, one gastroenteritis, and one lower respiratory infection. There were no hospital admissions. As a result of the review of drug-induced side effects, nine patients (Table 3 ) underwent a treatment change. Two patients required repeat bone densitometry and one patient with osteoporosis was referred to a bone specialist.

Table 2 The medication used by the reviewed patients

\begin{tabular}{lc}
\hline Drugs & Number \\
\hline Pred + AZA & 12 \\
Pred + CYA & 9 \\
Pred + MYC & 3 \\
Pred + AZA + CYA & 1 \\
Pred + CYA + MYC & 2 \\
AZA & 3 \\
CYA & 1 \\
MYC & 2 \\
AZA + CYA & 2 \\
CYA + MYC & 1 \\
\hline
\end{tabular}

Pred $=$ prednisolone; $\mathrm{AZA}=$ azathioprine $\quad \mathrm{CYA}=$ ciclosporin $\quad \mathrm{MYC}=$ mycophenolate.

Table 3 Reasons for change in treatment in 10 patients following health review

\begin{tabular}{|c|c|}
\hline Problems & Action \\
\hline $\begin{array}{l}\text { Ciclosporin toxicity (gingivitis, } \\
\text { hirsutism) }\end{array}$ & Reduce CYA dose \\
\hline Ciclosporin toxicity (hirsutism) & Change CYA to AZA \\
\hline Ciclosporin toxicity (gingivitis) & Reduce CYA dose \\
\hline $\begin{array}{l}\text { Ciclosporin toxicity (peripheral } \\
\text { neuropathy) }\end{array}$ & Change CYA to AZA \\
\hline $\begin{array}{l}\text { Steroid- and ciclosporin-induced } \\
\text { acne, hirsutism }\end{array}$ & $\begin{array}{l}\text { Reduce steroid and CYA } \\
\text { dose }\end{array}$ \\
\hline Steroid-induced acne, insomnia & Reduce steroid dose \\
\hline Steroid-induced mood swings & $\begin{array}{l}\text { Reduce steroid, increase } \\
\text { AZA dose }\end{array}$ \\
\hline Steroid-induced hirsutism & Reduce steroid dose \\
\hline $\begin{array}{l}\text { Mycophenolate gastrointestinal } \\
\text { symptoms }\end{array}$ & Change MYC to AZA \\
\hline
\end{tabular}

$\mathrm{AZA}=$ azathioprine $\mathrm{CYA}=$ ciclosporin; $\mathrm{MYC}=$ mycophenolate 
In addition to treatment changes made because of medication-induced side effects, some changes were made because of suboptimal treatment efficacy. The target visual acuity was felt to be maintained in 34 patients $(92 \%)$, the remaining three having mycophenolate added to their regime (2), or cataract extraction (1). The intraocular inflammation was considered to be adequately controlled in 31 patients (84\%), four of the remaining six requiring additional oral immunosuppression (two azathioprine, two mycophenolate) and the remaining two, additional topical steroid. There was some overlap between these two categories.

\section{Discussion}

The safe management of patients with sight-threatening ocular inflammation using oral immunosuppressives and steroids is complex, ${ }^{1,2}$ and the use of management protocols is recommended, as it has been for similar scenarios including renal transplantation. ${ }^{3}$ However, strict adherence to detailed protocols may be difficult to guarantee in a clinic with several staff including trainee doctors. We introduced this system of formal health review to enhance drug safety and to give patients a full opportunity to discuss management concerns and treatment side effects, thereby ensuring full involvement in management.

Several drug interaction risks may affect patients with intraocular inflammatory disease. The concurrent use of oral steroid and non-steroidal anti-inflammatory drugs increases the risk of dyspepsia and peptic ulceration. Drug absorption may be affected; for example, ciclosporin absorption may be increased by nifedipine, or decreased by phenytoin; steroid efficacy is greatly reduced by rifampicin; azathioprine effect is increased by allopurinol; and mycophenolate mofetil effect is reduced by antacids. Other physicians may commence new drug treatment, and FHR has reassured us that interactions are not missed.

Questioning revealed hitherto unreported steroid- or ciclosporin-induced skin and hair changes including striae and hirsutism in women, and acne in men. Such symptoms may not be readily volunteered, and facial hirsutism in women is often self-treated by waxing. The FHR gave greater opportunity for patients to discuss their concerns about side effects, and in some cases treatment changes were made.

The risk of steroid-induced bone loss is wellestablished and has been reported in uveitis. ${ }^{4}$ It is this clinic's policy to arrange densitometry within 3-6 months of commencing long-term steroid therapy, and to repeat every 2-3 years. The use of bone protective medication was checked and changed if necessary. The careful re-examination of bone densitometry results and an appraisal of non-iatrogenic risks for bone loss reassured us that our protocols for bone protection in steroid therapy were secure, and only one patient required review by a bone metabolism specialist.

Patients with occlusive retinal vasculitis, and some with Behçet's disease, carry intrinsic cardiovascular risks. ${ }^{5}$ Patients using steroid or ciclosporin may also be at increased cardiovascular risk because of induced hypertension, weight gain, or hyperlipidaemia. Coronary risk in our patients was assessed using body mass index, blood pressure, lipid profile, and diabetic status. While no patients was found to be at high cardiovascular risk, two with systemic hypertension required a review of treatment.

Oral immunosuppression carries a small but significant risk of dermatological or lymphatic cancer. This is well-recognised for those patients following renal transplantation, for whom a surveillance programme may be used. ${ }^{6}$ Although the risk of cancer in patients with intraocular inflammation rather than systemic inflammatory disease or after organ transplantation appears to be significantly less, we have raised the issue as part of this FHR and have provided information for patients to read at home. We feel that this has been an important development which, sensitively introduced, has not raised undue concern among our patients.

The task of comprehensive health review is timeconsuming, and approximately $30 \mathrm{~min}$ per patient per session are needed. However, for each patient requiring long-term immunosuppression, we do not feel that reassessment needs to be frequent. It is currently used as a single episode of monitoring to raise management issues for the supervising consultant, and this itself provokes closer attention to treatment-induced side effects within the normal clinic environment. We are confident that the ability to identify and manage problems that do not normally surface in medical consultation, justifies its usage.

In conclusion, we have found this system of formal health review to be useful to reassure us that our management protocols for the safe management of oral immunosuppression are largely effective. We feel that patient safety has been enhanced and a modified form of the review is to become a standard part of our patient management.

\section{References}

1 Stanbury RM, Graham EM. Systemic corticosteroid therapy - side effects and their management. $\mathrm{Br} \mathrm{J}$ Ophthalmol 1998; 82: 704-708.

2 Tamesis RR, Rodriguez A, Christen WG, Akova YA, Messmer E, Foster CS. Systemic drug toxicity trends in immunosuppressive therapy of immune and inflammatory ocular disease. Ophthalmology 1996; 103: 768-775. 
3 Cohen D, Galbraith C. General health management and longterm care of the renal transplant recipient. Am J Kidney Dis 2001; 38: S10-S24.

4 Jones NP, Anderton LC, Cheong FM, Whallett A, Stanford MR, Murray PI et al. Corticosteroid-induced osteoporosis in patients with uveitis. Eye 2002; 16: 587-593.
5 Palmer HE, Zaman AG, Edelsten CE, Stanford MR, Graham EM. Systemic morbidity in patients with isolated idiopathic retinal vasculitis. Lancet 1995; 346: 505-506.

6 Reece SM, Harden PN, Smith AG, Ramsay HM. A model for nurse-led skin cancer surveillance following renal transplantation. Nephrol Nurs J 2002; 29: 257-259.

Supplementary Information accompanies the paper on Eye website (http://www.nature.com/eye) 